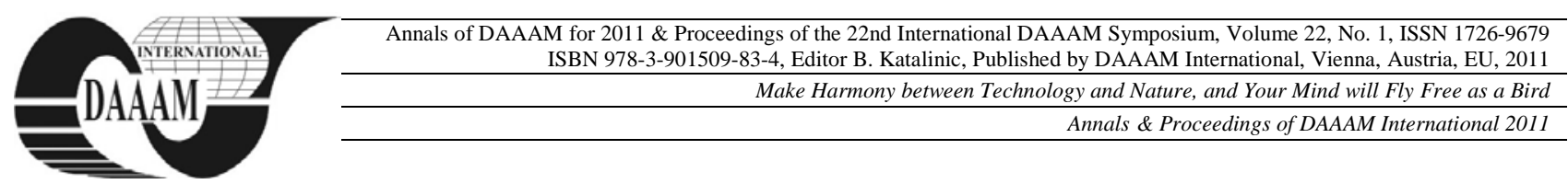

\title{
NEURAL NETWORKS FOR SMART HOMES AND ENERGY-EFFICIENCY
}

\author{
TEICH, T[obias]; ROESSLER, F[alko]; SZENDREI, D[anny] \& FRANKE, S[usan]
}

\begin{abstract}
In a project to reduce energy consumption, the use of technology which helps metering and controlling lifestyle effects is essential. Smart meters and intelligent systems that contribute to environmental arwareness enable private homeowners or tenants to see and actively control their cost of lifestyle. As a part of Smart Home systems neural networks are considered to be of assistance for user-based systems and consumption prediction. The observation of collected data over a period of time offers many opportunities to disvocer potential applications that help optimizing specific tasks. Controlling the target temperature at a specific time of day, based on the habits and preferences of a tenant is one first chosen way to make daily life easier and at the same time make it possible to design Smart homes that compromise between energy-efficieny and personal comfort. For that purpose a neural network is designed and tested under varying premises. The results are promising and the insights will enable future works in following projects.
\end{abstract}

Key words: neural networks, learning, energy-efficiency, neural control

\section{INTRODUCTION}

This paper contributes to a research project at UAS Zwickau in collaboration with a communal housing association. Various sensors in newly refurbished tenements capture data of energy consumption at radiators and bus bars as well as air quality, temperature and weather conditions (Teich et al., 2010). At the present time tenants have the possibility to adjust the desired target temperature for every single room via a central touch panel that can also be timed beforehand. These settings have to be changed as specific conditions alter. There are data which prove that settings for different time had been changed once or even several times a day. The objective for the current research is how to train and implement neural networks that can assist users in setting temperature profiles. As one natural adaptation the data can be used to train a neural network on the tenant's preferences that then will automatically adjust the target temperature regarding the data.

\section{NEURAL NETWORKS}

Biologically inspired procedures such as genetic algorithms, ant colony optimization and neural networks (Kramer, 2009) apply to areas where traditional mathematical methods can not be realized due to the fact that there are not enough resources to find an exact solution. These procedures mimic the way of cooperation and information processing that exists in nature. With (artificial) neural networks we have one abstract method comparable to the processing capabilities of the human brain. In practice they are being used as subsystems in algorithmic frameworks since they are not suitable to provide an overall system (Deco, Schuermann, 2001). Yet they can perform complex tasks ranging from recognition to short-term projection.

Neural networks consist of a minimum of two layers of neurons which each has an input, a body and an output part. In case there are only two layers, the first, also called input layer will get the input directly from external data, so there is no input connection from other neurons. A neuron will generate an output level from its total activation, i.e. the sum of net input which is then forwarded to the connected neurons in the next layer. The output level may be kept the same value as input for the following neurons, though a multiplication with a variable is usually the case. There can be several layers between the first and last one, so called hidden layers which fulfill different functions. Neurons in the last layer are called output neurons. Their activation level represents the result of the 'thinking' process. They usually do not have an output connection to another unit or layer. Neurons of the hidden layers are also called hidden units which can have many different functions and characteristics, for instance they can be used as a form of short-term memory buffer which enables the network to make better predictions in case there are reappearing patterns.

In detail there are many different types of neural networks (Haykin, 1994) which are not necessarily restricted to one specific executable function. Teaching a net is possible in different ways (Zell, 2000) which are in general divided in methods of supervised and unsupervised learning.

\section{DATA SELECTION AND TRAINING}

Before presenting training data to a neural network, the scenario of required application has to be well considered. Data are collected from sensors in the building and the outside environment. The reference Smart Homes have been equipped with a KNX bus system on which basis most of today's standard sensors communicate. Sensor data are sent to a local facility server in adjustable cycles. Programmed algorithms retrieve these data to a second central server over night. From that point on, we have to use procedures to filter and to preprocess the data basis (Teich, Zimmermann, 2010).

Depending on the intended result we have to evaluate which data are required. In some cases it does make sense to create artificial data, for instance as harmonizing factors. Preprocessing is also necessary since the neurons work with numeric data that are then processed and forwarded. Neural networks possess the feature that they are able to handle deviant or even missing data, thus, show some sort of stability.

Results of training can be evaluated by a set of test data. Selection of data for training as well as for test has to be made very careful. If they are nor suited or do not represent similar variety the net is not able to produce reasonable output. The first models used a six dimensional input vector containing data of temperatures, time and weather conditions over a time frame of two months within heating period. A time variant neural network with multiple hidden layers already showed good results after a short training period, as being shown in fig. 1 in which the dark line represents the target output of the output neuron while the brighter line shows the current level of the trained net. Deviations from the target output are minimal and can be explained by the altering sensibility of a person in regard to their temperature preference depending on physical conditions and other factors. 
Our experiments were made with the freeware tool MemBrain, a graphical neural networks editor and simulator by Thomas Jetter (Rey \& Wender, 2011; www.membrain-nn.de) which allows designing simple networks.

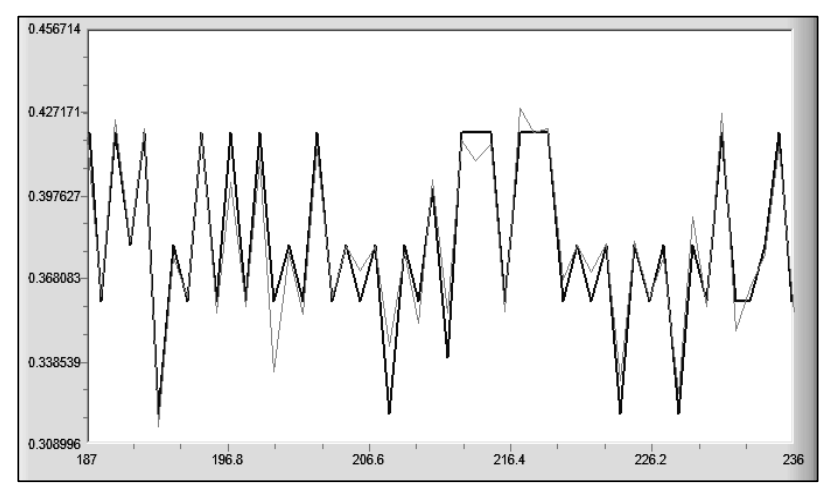

Fig. 1. Target and Current activation of the output neuron

\section{COURSE OF ACTION}

After individual tests there was always a step back to redesign the network and data as well as to find out which setup shows the best characteristics. If different tests had similar results the networks were analyzed in terms of performance and which is easier to implement into a system of middleware. Training a net was done with data containing two years measured four times per day. Due to the fact that no reliable real data exist for such a long period yet, they had to be generated within plausible intervals. For the time being it is planned to design neural networks to learn on short-term basis and extend training data as they arise. First tests showed that there is a need to add more dimensions to the input vector in order to have reliable output prediction under influence of seasonal specifics. It could be achieved that by adding new (artificial) parameters that were expected to harmonize transitions between seasons. It proved to be effective and even level areas which before had greater deviations as shown in fig. 2. Under the assumption that the target temperature will not be set below a certain limit, for instance 15 degrees Celsius in the summer season, because it has only meaning in situations heating is needed. A subsequent decision was to disregard nonheating seasons since they affect the learning success for the whole set of data. Experiments with additional data showed different results, yet a final commitment has still to be made.

For this kind of scenario more than only one possible solution was found as a design for a neural network. A simple recurrent network (SRN) proved to be just as effective as feedforward networks, yet the choice of an appropriate activation function - for details (Lippe, 2006) - depends on the number of dimensions as well as output data requirements. While the training results for two-month data were quite similar for the output neuron activation function of the types 'tangens hyperbolicus' and 'identical' the net showed better performance in terms of speed and accuracy with the latter activation function when learning higher dimensional input vectors and a higher quantity of data. When adding new rooms to the system it will multiply the total data volume by the count which then will also prolong the required training time, yet it still is within an acceptable time frame. Re-training a net will be done only if there are greater divergences over a certain period. Final decisions about whether the net should be trained on a shortterm or rather long-term basis have to be made after respective research. New approaches and possible solutions are examined and will be taken into consideration as well.

The work and research for the time being is limited to small easy-to-use applications within Smart Home projects. The (sub) systems will play a supporting role for energy saving concepts until further opportunities unveil.

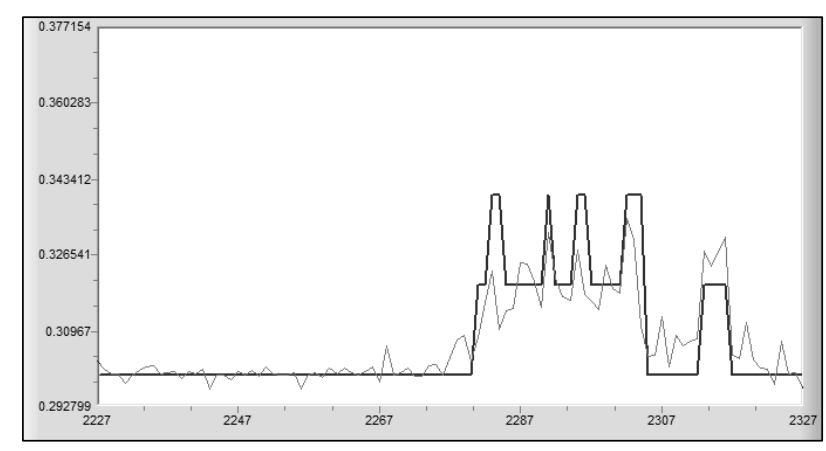

Fig. 2. Target and current output data - summer season

\section{RESULTS AND FURTHER STEPS}

Neural networks enable the construction of various predictive systems as well as analyzing the effect of different data in specific situations. Still, retracing the neural processes is not easy, due to the varios, complex interconnections in between. It is considered the target temperature scenario to be the first one implemented within the next months and further research will be conducted in order to find other useful applications for neural networks in Smart Homes. Another realistic scenario is air quality control. Therefor, sensors have been installed in the tenements. These sensors measure CO2concentration, temperature and air moisture. For the time being it is a very promising approach for different situations where 'learning' is possible or even required in the first place. Neural networks may become one essential element in building Smart Homes, be it for the reasons of personalized services and the ability to adapt to new situations, e.g. different or multiple users. The author's work is still in progress as there are many tasks in the field of collecting data in easier ways. Automatic procedures to retrieve data and train neural networks in case changes are needed will be of much use for later real-time applications. At the present time work is done on how to connect the system with reliable both internal sensor data as well as external data sources, for instance from meteorological service providers. Test runs are going to be done for our first model under real conditions in one tenement for different rooms as soon as these tasks are finished. Depending on the results, the neural processes have to optimized or aligned.

\section{REFERENCES}

Deco, G., Schuermann, B. (2001), Information Dynamics: Foundations and Applications, Springer, ISBN 0-38795047-8, Berlin Heidelberg, Germany

Haykin, S. (1994). Neural Networks: A Comprehensive Foundation 1st, Prentice Hall PTR, ISBN 0-023-52761-7, Upper Saddle River, NJ, USA

Kramer, O. (2009). Computational Intelligence, SpringerVerlag, K., ISBN 978-3-540-79738-8, Berlin Heidelberg, Germany

Lippe, W. (2006). Soft-Computing mit Neuronalen Netzen, Fuzzy-Logic und Evolutionaeren Algorithmen, Lippe, W., ISBN-10 3-540-20972-7, Springer-Verlag Berlin Heidelberg, Germany

Rey, G., Wender, K. (2011). Neuronale Netze: Eine Einfuehrung in die Grundlagen, Anwendungen und Datenauswertung, Verlag Hans Huber, ISBN 987-3-45684881-5, Bern, Germany

Teich, T., Zimmermann, M. and Other (2010). Intelligent Building Automation. In Karras, D.A., Moustafa K.A.F., Tang, D. (ed.), International Conference on Automation, Robotics and Control Systems. 53 - 57. ISRST. Orlando, Florida.

Zell, A. (2000). Simulation neuronaler Netze. Oldenbourg Wissenschaftsverlag, ISBN 3-486-24350-0, Oldenbourg, Germany 